\section{AB0451 TO EVALUATE THE PREVALENCE OF INTERSTITIAL LUNG DISEASE (ILD) AND/OR PULMONARY ARTERIAL HYPERTENSION (PAH) IN PATIENTS AFFECTED BY SYSTEMIC SCLEROSIS (SSC) AND TO DETERMINE THE FACTORS ASSOCIATED WITH ILD}

R. Ortega Castro ${ }^{1}$, R. Mariscal-Ocaña ${ }^{2}$, M. Rojas-Giménez ${ }^{1}$, J. Calvo Gutierrez ${ }^{1}$, A. Escudero Contreras ${ }^{1}$, C. López-Medina ${ }^{1}{ }^{1}$ Reina Sofia Universitary Hospital/Maimonides Institute of Biomedical Research of Córdoba (IMIBIC), University of Cordoba, Clinical Management Unit of Rheumatology, Córdoba, Spain; ${ }^{2}$ Reina Sofia Universitary Hospital/University of Cordoba, Clinical Management Unit of Rheumatology, Córdoba, Spain

Background: Systemic sclerosis (SSc) is a chronic autoimmune disease that carries significant mortality. Despite diagnostic and therapeutic advances in recent years, there is still a significant percentage of patients who do not present a complete clinical response, with the associated increase in morbidity and mortality. Specifically, pulmonary disease is frequent and entails a poor prognosis, with interstitial lung disease (ILD) and pulmonary hypertension (PAH) being the two most important complications, the first and second cause of mortality, respectively.

Objectives: To evaluate the prevalence of ILD and/or PAH in patients affected by $\mathrm{SSc}$ and to determine the factors associated with ILD.

Methods: Cross-sectional observational study of 102 patients diagnosed with SSc (Limited, Diffuse, SSc without scleroderma or Pre-scleroderma), treated between 1975 and 2020 at the Reina Sofia University Hospital in Cordoba. A descriptive study of the cohort was carried out and factors independently associated with ILD were evaluated using a multiple logistic regression model.

Results: 102 patients were included, $87.3 \%$ of these were female with an average age of 50.8 (14) years. There were 20 deaths (19.8\%), from which $55 \%$ died because of SSc and the main reason was ILD and/or PAH. Respiratory complications (as ILD or as PAH) were present in 59 patients (57.8\%), of whom 52 were diagnosed with ILD (90.4\% with a pattern of non-specific interstitial pneumonia) and $25 \mathrm{PAH}$, whose mean pulmonary artery systolic pressure was 47.16 (18.54) $\mathrm{mmHg}$. Anti-topoisomerase I antibodies were positive in $34.6 \%$ of patients who developed ILD, while anticentromere antibodies were more frequent in SSc without interstitial lung disease (80\%). Independent factors associated with ILD were type of SSc, proximal skin involvement, anticentromere antibodies, current treatment with corticoids and the death.

Conclusion: Just over half of the patients with SSc have lung disease (as ILD or as PAH). The main risk factors associated with ILD are proximal skin involvement and treatment with glucocorticoids, probably in the context of more severe forms that require more treatment. Anticentromere antibodies are more prevalent in patients with Limited SSc and their expression decreases the risk of developing ILD in these patients.

REFERENCES:

[1] Orlandi M, Barsotti S, Lepri G, et al. Clin Exp Rheumatol 2018 Jul-Aug; 36 Suppl 113: 3-23

[2] Hao Y, Hudson M, Baron M, et al. Arthritis Rheumatol. 2017;69(5):1067-1077.

[3] Furue M, Mitoma C, Mitoma H, et al. Immunol Res. 2017 Aug; 65: 790-7.

[4] Nihtyanova SI, Schreiber BE, Ong VH, et al. Arthritis Rheumatol. 2014 Jun; 66: $1625-35$

Disclosure of Interests: None declared.

DOI: 10.1136/annrheumdis-2021-eular.3524

\section{AB0452 GENDER FEATURES IN PSYCHOLOGICAL STATUS IN PATIENTS WITH LUNG ULTRASOUND SYNDROME}

N. Shamsutdinova ${ }^{1}$, D. Abdulganieva ${ }^{2}$, E. Kirillova ${ }^{2}$, D. Gaisina ${ }^{1}$, A. Fatikhova ${ }^{1}$. ${ }^{1}$ Kazan State Medical Universlty, Hospital Therapy, Казань, Russian Federation; ${ }^{2}$ Kazan State Medical Universlty, Hospital Therapy, Kazan, Russian Federation

Background: The use of lung ultrasound (LUS) in the management of patients with ILD has the advantage of lower cost and the absence of $x$-ray radiation.

Objectives: to establish the relationship between ultrasound manifestations of interstitial syndrome and indicators of psychological status in patients with secondary ILD in patients with rheumatic diseases.

Methods: 25 patients with secondary ILD in patients with rheumatic diseases (15 patients with systemic scleroderma, 8 patients with ANCA associated systemic vasculitis, 2 patients with dermatomyositis) were investigated. All patients underwent a standard clinical examination, high-resolution RCT of lungs. To assess depression and anxiety were used the following scales: State-Trait Anxiety Inventory, symptomatic questionnaire SCL-90-R, Beck Depression Inventory. LUS was carried out for the evaluation of the location and number of B-lines on both right and left hemithoraces using commercially available echographic equipment with a 5-12 MHz linear transducer (Accuvix A30, Samsung Medison).

Results: Most patients had an average number of B-lines 24,5[11,5;34,0]. In woman the total number of B-lines correlated with the situational anxiety $(r=-0,71, p<0,05)$, with the personal anxiety $(r=-0,6, p<0,05)$ and with somatization $(r=-0,67, p<0,05)$. Also there was a trend between somatic $(r=-0,49, p=0,08)$ and affective $(r=-0,51, p=0,07)$ subscales, general depression $(r=-0,48, p=0,1)$ but did not achieve statistical significance, probably due to the small number of patients. Among patients, including men and women, no statistically significant correlation was found.

Conclusion: The relationship between situational, personal anxiety, somatization and the total number of B-linesamong women with secondary ILDwas found. More research is needed to clarify details.

\section{REFERENCES:}

[1] Dietrich CF, Mathis G, Blaivas M, Volpicelli G, Seibel A, Wastl D, Atkinson NS, Cui XW, Fan M, Yi D. Lung B-line artefacts and their use. J Thorac Dis 2016;8(6):1356-1365. doi: 10.21037/jtd.2016.04.55

[2] Tatiana Barskova, Luna Gargani, Serena Guiducci, et al. Lung ultrasound fo the screening of interstitial lung disease in very early systemic sclerosis Ann Rheum Dis 2013;72:390-395. doi:10.1136/annrheumdis-2011-201072

Disclosure of Interests: None declared.

DOI: 10.1136/annrheumdis-2021-eular.3546

\section{AB0453 THE CHARACTERISTICS OF SSC-ILD PATIENTS WITH ACTIVE THERAPY IN DIFFERENT PROGNOSIS INTERVALS BASED ON DISCRIMINANT ANALYSIS}

O. Ovsyannikova ${ }^{1}$, L. Garzanova ${ }^{1}$, L. P. Ananyeva ${ }^{1}$, O. Koneva ${ }^{1}$, S. Glukhova ${ }^{2}$ O. Desinova ${ }^{1}$, M. Starovoytova ${ }^{1}$, R. Shayakhmetova ${ }^{1} .{ }^{1}$ VA Nasonova Research Institute of Rheumatology, Laboratory of microcirculation and inflammation, Moscow, Russian Federation; ${ }^{2}$ VA Nasonova Research Institute of Rheumatology, Educational and methodical department, Moscow, Russian Federation

Background: Interstitial lung disease (ILD) frequently complicates SSc and can be a debilitating disorder with a poor prognosis. Several risk factors for progressive SSc-ILD have been identified and prognostic scoring systems have identified predictors of mortality. It's important to develop treatment recommendation. Objectives: To use the discrimination function for pts with SSc-ILD and highlight pts with different prognosis.

Methods: It was a longitudinal study involving 140pts with SSc-ILD. The mean age was $46.7 \pm 13.9 y s$, females $82 \%$, SSc duration $6.2 \pm 5.8 y$ s, diff subset- $54 \%$. The mean duration of follow up was $73,2 \pm 27,8$ months. All pts received low- and moderate-dose glucocorticoids (Gc), RTM (the mean dose $3,14 \pm 2,38 \mathrm{~g}$ ) and 77 pts had received parenteral CyP(mean dose $15,9 \pm 15,6 \mathrm{~g}$ ).

We have got the discrimination function that included: ground glass opacities (GGO)(1-the absents of GGO; 2-the presents of GGO); index EScSG(the digital value); $F V C(F V C \geq 75=0, F V C<75=1$ ); the maximum daily dose of $G c$ (the digital value,mg); Gamma globulins (the digital value,\%); cyclophosphamide (CYC) (the absence of $\mathrm{CyP}=0$, the treatment of $\mathrm{CYC}=1)$; $\mathrm{DLCo}(\mathrm{DLCO}<52 \%=0, \mathrm{DLCO} \geq 52=1$ ). We assessed all parameters at the entry in the study except DLco evaluated after one year of follow up. Based on this analysis the equation was developed. The equation of prognosis is $3.14 \mathrm{GGO}+0,70$ index EScSG-1,326 FVC- 0,1 the maximum daily dose of glucocorticoids+0,136 Gamma globulins +1.066 CYP-1.075DLCO $\leq 5,8$.

Value of equation of prognosis to 5.8 corresponds to stabilization or good prognosis and value after 5.8 corresponds to poor prognosis.

Results: On results of discrimination function pts were distributed in the following order: 22 pts(16\%) were into zone of stabilization(Group 1) and $118(84 \%)$ in zone of poor prognosis, among which 13pts died during the study. That's why pts in zone of poor prognosis were divided into group 2 - survived pts $(n=105(75 \%))$ and group $3-$ died pts $(n=13(9 \%))$.So we have got 3groups; the age, SSc duration, gender proportion and SSc forms, were similar in the all groups $(p>0,05)$.

Table 1. Baseline characteristics of the groups

\begin{tabular}{lccc}
\hline Parameters & $\begin{array}{c}\text { Group } 1(\mathrm{n}=22) \\
\mathrm{M} \pm \mathrm{SD}\end{array}$ & $\begin{array}{c}\text { Group 2 }(\mathrm{n}=105) \\
\mathrm{M} \pm \mathrm{SD}\end{array}$ & $\begin{array}{c}\text { Group 3 }(\mathrm{n}=13) \\
\mathrm{M} \pm \mathrm{SD}\end{array}$ \\
\hline CRP mg/ml & $9,4 \pm 10,4$ & $11,5 \pm 23,4$ & $21,5 \pm 27,1$ \\
ME [25\%/75\%] & $7,5[1,9 ; 11,2]$ & $5[1,6 ; 10,4]$ & $9,9[5 ; 32,8]$ \\
ESR mm/h & $17 \pm 16,35$ & $23,6 \pm 17$ & $22,9 \pm 14,3$ \\
a-Scl-70 u/ml & $62,5 \pm 85^{\star}$ & $118,6 \pm 93,09^{\star}$ & $102,1 \pm 96$ \\
Skin score & $9,63 \pm 7,7$ & $11,5 \pm 9,9$ & $9 \pm 9,23$ \\
Index EScSG & $1,9 \pm 1,7^{\star *}$ & $3,4 \pm 1,9^{* *}$ & $2,9 \pm 1,1$ \\
Mean PAP mm hg & $31,9 \pm 7 \beta \beta \beta$ & $35 \pm 11,9 \beta$ & $47,5 \pm 23,3 \beta \beta$ \\
Cumulative dose of CyP g & $1,5 \pm 3 \gamma \gamma \gamma$ & $11,4 \pm 14,4 \gamma$ & $18,9 \pm 23,3 \gamma \gamma$ \\
ME [25\%/75\%] & $0[0 ; 2]$ & $4,9[0 ; 17]$ & $4[0 ; 46,5]$ \\
Cumulative dose of RTM, g & $3,15 \pm 1,6$ & $2,63 \pm 1,36$ & $2,2 \pm 1,5$ \\
\hline
\end{tabular}

* $-\mathrm{p}=0,01 ;{ }^{* *} \mathrm{p}=0,0008 ; \beta-\mathrm{p}=0,006 ; \beta \beta-\mathrm{p}=0,03 ; \gamma-\mathrm{p}=0,002 ; \gamma \gamma-\mathrm{p}=0,0018$ Mean level a-Scl-70, Index EScSG were significantly lower in group 1 vs group 2 . Mean value of PAP and dose of CyP were significantly increased in group 3 . It should be noted that pts with good prognosis have had low level of all parameters and high dose of RTM. 
Conclusion: The discrimination function is a proposed screening algorithm for pts with SSc-ILD who could develop progression disease. Usage of discrimination function revealed that $84 \%$ pts with ILD-SSc have got poor prognosis and $9 \%$ of them have got fatal outcome. Usage the equation of prognosis is very useful to choice of treatment and observation pts SSc-ILD.

Disclosure of Interests: None declared.

DOI: 10.1136/annrheumdis-2021-eular.3589

\section{AB0454 SPECIFICITY OF INTERSTITIAL LUNG DISEASE IN PATIENTS WITH SYSTEMIC SCLEROSIS POSITIVE FOR A-TOPO-1 AND A-RNP}

R. Shayakhmetova ${ }^{1}$, L. P. Ananyeva ${ }^{1}$, O. Koneva ${ }^{1}$, M. Starovoytova ${ }^{1}$,

O. Desinova', O. Ovsyannikova ${ }^{1}$, L. Garzanova', A. Khelkovskaia-Sergeeva ${ }^{1}$,

S. Glukhova' ${ }^{1}$ V.A. Nasonova Research Institute of Rheumatology; V.A.

Nasonova Research Institute of Rheumatology, Moscow, Russian Federation

Background: The classification criteria of SSC (EULAR/ACR 2013) compared to the criteria of 1980 showed high sensitivity and allow to detect the full spectrum of systemic sclerosis (SSc). The use of the new criteria led to the fact that some of the patients who were diagnosed with MCTD began to meet the criteria of SSc. Since these patients have hyperproduction of antibodies to RNP, but no other SSc-specific autoantibodies, we believe that they represent a special clinical and immunological phenotype of SSc. Lung involvement in SSc impaires the quality of life and the prognosis, so the study of interstitial lung disease(ILD) in different phenotypes is relevant.

Objectives: To compare the main manifestations of ILD in two groups of patients who meet the classification criteria of SSc 2013 - positive for a-Topo-1 and for a-RNP.

Methods: The study included 100 patients. The first group - 50 patients positive for a-Topo-1 (45 women and 5 men, mean age $55 \pm 10$ years, duration of the disease $10.2 \pm 8$ years, subset of the disease: diffuse $52 \%$, limited $48 \%$ ) and the second group - 50 patients positive for a-RNP ( 43 women and 7 men, mean age $44.5 \pm 14$ years, duration of the disease $11.7 \pm 8$ years; form of the disease: diffuse $14 \%$, limited 86 ). The diagnosis of ILD was established by HRCT.

Results: In patients of group I ILD was detected in $94 \%$ of cases, in group II - $68 \%(p<0.05)$. In both groups a decrease in functional lung tests was noted with a high frequency, but a severe decrease in the lung diffusion capacity was significantly more often observed in group I.

Table 1. Data of laboratory and instrumental parameters

\begin{tabular}{lccc}
\hline & a-Topo-1- positive & a-RNP-positive & \\
Parameters & $\mathrm{n}(\%)$ & $\mathrm{n}(\%)$ & $\mathrm{p}$ \\
\hline Decrease of DLCO $<80 \%$ & $49(98)$ & $43(86)$ & $\mathrm{p}<0,05$ \\
DLCO $<55 \%$, & $33(66)$ & $16(32)$ & $\mathrm{p}<0,05$ \\
Decrease of FVC $<80 \%$ & $19(38)$ & $10(20)$ & $\mathrm{p}<0,05$ \\
FVC $<70 \%$ & $13(26)$ & $7(14)$ & $\mathrm{NS}$ \\
Ground glass opacity & $25(50)$ & $26(52)$ & $\mathrm{NS}$ \\
Honeycombing & $17(34)$ & $8(16)$ & $\mathrm{p}<0,05$ \\
SPAP $>40 \mathrm{~mm} . \mathrm{Hg}$, & $6(12)$ & $10(20)$ & $\mathrm{NS}$ \\
including PAH & $2(4)$ & $3(4,6)$ & $\mathrm{NS}$ \\
ANA $>1: 640$ & $37(74)$ & $45(90)$ & $\mathrm{p}<0,05$ \\
\hline
\end{tabular}

* ANA - antinuclear antibodies, PAH - pulmonary arterial hypertension, SPAP - systolic pulmonary artery pressure ${ }^{* *}$ confirmed by the data of catheterization of the right heart

Typical changes according to HRCT were detected in both groups, but fibrotic changes dominates in group I. There were no significant differences in the frequency of occurrence of PAH. Patients of group II had high level of ANA more often.

Conclusion: In both groups, a high frequency and similar manifestations of ILD were noted. In patients with SSc positive for anti-U1RNP, despite the predominance of limited skin lesions (in $86 \%$ of patients), a high incidence of ILD was detected, while the frequency of PAH was comparable to SSc in general. Compared with a-Topo-1 positive patients, ILD was significantly less frequent in anti-U1RNP positive patients and was accompanied with a smaller reduction of DLCO and/or FVC and a lower frequency of severe lung fibrosis. These data confirm the similarity of lung involvement in compared phenotypes of SSc and indicate the importance for screening for ILD in patients with hyperproduction of a-RNP, especially at an early stage of the disease.

REFERENCES:

[1] Van den Hoogen F, Khanna D, Fransen J, et al. 2013 classification criteria for systemic sclerosis: an American College of Rheumatology/European League Against Rheumatism collaborative initiative. Ann Rheum Dis 2013;72:1747-55

Disclosure of Interests: None declared.

DOI: 10.1136/annrheumdis-2021-eular.3591

\section{AB0455 \\ MODIFICATION OF PROGNOSIS INTERVALS WAS DERIVED BY THE DISCRIMINATION FUNCTION FOR SSC-ILD PATIENTS}

O. Ovsyannikova ${ }^{1}$, L. P. Ananyeva ${ }^{1}$, L. Garzanova ${ }^{1}$, O. Koneva ${ }^{2}$, S. Glukhova ${ }^{2}$, M. Starovoytova ${ }^{1}$, O. Desinova ${ }^{1}$, R. Shayakhmetova ${ }^{1} .{ }^{1} V A$ Nasonova Research Institute of Rheumatology, Laboratory of Microcirculation and Inflammation, Moscow, Russian Federation; ${ }^{2}$ VA Nasonova Research Institute of Rheumatology, Educational and Methodical Department, Moscow, Russian Federation

Background: Systemic sclerosis (SSc) is a multisystem autoimmune disease Pulmonary alterations are the major cause of mortality in SSc. SSc-ILD has heterogeneous disease progression: many patients will have a chronic, indolent course while others may develop progressive, life-threatening disease.

Objectives: To assess prognosis intervals for pts with SSc-ILD.

Methods: It was a longitudinal study involving 140 pts with SSc-ILD. The mean age was $46.7 \pm 13.9$ years, females $82 \%$, SSc duration $6.2 \pm 5.8$ years, diffuse subset - $54 \%$. The mean duration of follow up was $73,2 \pm 27,8$ months. Clinical, laboratory, and immunologic data were collected on all patients.

Based on analysis of discrimination function the equation of prognosis was developed. The equation is $3.14 \mathrm{GGO}$ (ground glass opacities) $+0,7$ index EScSG - 1,326 FVC - 0,1 the maximum daily dose of glucocorticoids + 0,136 Gamma globulins $+1.066 \mathrm{CYP}-1.075 \mathrm{DLCO} \leq 5,8$. Value of equation of prognosis to 5.8 corresponds to stabilization or good prognosis and value after 5.8 corresponds to poor prognosis. The equation of prognosis had $86 \%$ sensitivity and $56 \%$ specificity. The cut-off point 4.7 was represented $65 \%$ sensitivity and $67 \%$ specificity. The prognosis intervals were following: value of equation to 4.7 corresponds to good prognosis; value from 4.8 to 5.8 corresponds to stabilization and value after 5.8 corresponds to poor prognosis.

Results: Pts were distributed into groups depending on results of discrimination function: 14 pts (10\%) - zone of good prognosis; 8 pts $(6 \%)$ - zone of stabilization and $118(84 \%)$ - zone of poor prognosis. We have decided to join pts from zone good prognosis and zone stabilization into united group (U-group) - 22 pts $(16 \%)$

Table 1. Comparative characteristics of the groups

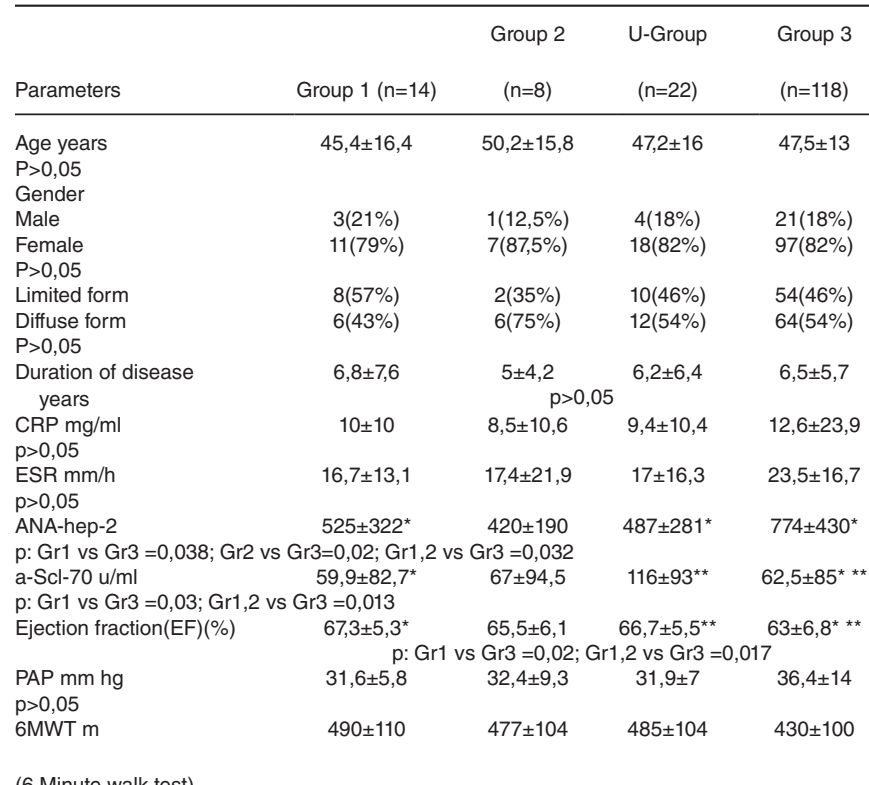

$p>0,05$

The age, SSc duration, gender proportion and SSc forms, were similar in the all groups $(p>0,05)$. In the study mean dates of ANF-hep-2, a-Scl-70 were significantly lower and EF was significantly higher in groups 1 and U-group than in group 3. In groups 1,2 and U-group the mean levels of ESR, CRP PAP were lower and value of $6 \mathrm{MWT}$ were higher than mean levels in group 3 accordingly. So separately group 2 didn't have significant differences with group 1 and 3 .

Conclusion: In the study we didn't find any correlation between meanings of group 2 and groups 1,3 but the meanings of group 1 and U-group have had the 